\title{
Genetic Drivers of Pancreatic Cancer Are Identical Between the Primary Tumor and a Secondary Lesion in a Long-Term ( $>5$ Years) Survivor After a Whipple Procedure
}

Tyler M. Bauer, ${ }^{1}$ Teena Dhir, ${ }^{2}$ Adam Strickland, ${ }^{2}$ Henry Thomsett, ${ }^{2}$ Austin B. Goetz, ${ }^{2}$

Shawnna Cannaday, Jonathan R. Brody, ${ }^{2}$ Michael J. Pishvaian, ${ }^{3}$ and Charles J. Yeo 2,*

\begin{abstract}
Background: A new mass in the remnant pancreas of a patient with previously resected pancreatic ductal adenocarcinoma (PDA) typically represents either a recurrence of the initial primary tumor or a second primary tumor. Recent advances in next-generation sequencing (NGS) strategies allow us to compare the genetic makeup of primary and secondary lesions.

Case presentation: A 50-year-old Caucasian female presented for a surgical evaluation of a new biopsy-proven PDA at the junction of the body and tail of the pancreas. Six years prior, in 2011, the patient was found to have a T3NOMO PDA of the pancreatic head, which was surgically resected with a classic Whipple procedure and concurrent hemicolectomy. Pathology showed pancreatic intraepithelial neoplasia grade 2 and PDA with negative surgical margins, positive perineural spread, and negative lymphovascular spread, and the patient received adjuvant chemotherapy and local radiation. In 2017, she was diagnosed with a new PDA lesion in the remaining pancreatic body far from the previous anastomosis site and was taken to surgery for a completion pancreatectomy and revision of the gastrojejunostomy. NGS was performed on both specimens. Both lesions shared identical mutations in KRAS, TP53, and CDKN2A genes. Amplifications of MYC and mutant KRAS were identified in the 2017 tumor and an ACVR1B mutation was identified in the 2011 tumor, but was not found in the 2017 tumor. Conclusions: This case demonstrates the ability to evaluate similarities between key genetic drivers from a resected primary tumor and a PDA lesion that presented in the same patient 6 years later. Histological analysis and NGS can be used to understand potential differences and similarities between lesions and may be useful in future studies as predictive markers or to provide insight into resistance mechanisms (e.g., MYC amplification).
\end{abstract}

Keywords: KRAS; metachronous; MYC; pancreatic ductal adenocarcinoma; pancreaticoduodenectomy; Whipple procedure

\section{Introduction}

Pancreatic ductal adenocarcinoma (PDA) accounts for 45,000 cancer diagnoses in the United States per year and $90-95 \%$ of all pancreatic cancers. ${ }^{1-3}$ PDA has historically high rates of recurrence and the lowest 5-year survival of any cancer. ${ }^{2}$ Only $20 \%$ of patients are eligible for resection upon initial presentation, ${ }^{4}$ with an average postresection survival of 16-24 months; 5-year survival postresection is $\sim 20-35 \%{ }^{5,6}$ Surgical candidates classically received adjuvant gemcitabine, although emerging data involving the use of adjuvant mFOLFIRINOX may be superior, with a median overall survival of $\sim 54$

\footnotetext{
${ }^{1}$ Sidney Kimmel Medical College, Thomas Jefferson University, Philadelphia, Pennsylvania.

${ }^{2}$ Department of Surgery, The Jefferson Pancreas, Biliary, and Related Cancer Center, Thomas Jefferson University, Philadelphia, Pennsylvania.

${ }^{3}$ Lombardi Comprehensive Cancer Center, Georgetown University, Washington, District of Columbia.

*Address correspondence to: Charles J. Yeo, MD, Department of Surgery, 1015 Walnut Street, Curtis 620, Thomas Jefferson University, Philadelphia, PA 19107, E-mail: charles.yeo@jefferson.edu
}

(c) Tyler M. Bauer et al. 2018; Published by Mary Ann Liebert, Inc. This Open Access article is distributed under the terms of the Creative Commons License (http://creativecommons.org/licenses/by/4.0), which permits unrestricted use, distribution, and reproduction in any medium, provided the original work is properly cited. 
months. ${ }^{7}$ Predictors of poor survival include positive surgical margins, positive lymph node metastasis, large tumor size $(>3 \mathrm{~cm})$, and poor histological differentiation. ${ }^{5,8}$ An unfortunate reality of treating pancreatic cancer is that even after 5 years of disease-free survival, recurrent disease is still possible, with $\sim 20 \%$ of patients who survive 5 years having recurrences (both locoregional and distant). ${ }^{6}$ Resection of locally recurrent lesions is safe and effective in selected individuals., ${ }^{9,10}$

To fully understand this disease, it is important to understand PDA's biology, including key driver mutations leading to the progression of normal pancreatic tissue to PDA. These driver mutations include mutations in KRAS and TP53 genes, with over 95\% of PDA cases having a KRAS mutation (G12D most common). ${ }^{11}$ There is a clear link between KRAS mutations and inactivation of important tumor suppressors, such as CDKN2A and TP53, which causes the progression of pancreatic intraepithelial neoplasia (PanIN) lesions to PDA; 90\% of low-grade PanIN-1A lesions have a KRAS mutation, which is evidence that it is a driver mutation at an early stage in PDA. ${ }^{12}$ After progression to PanIN-1B, $95 \%$ of lesions acquire a mutation in CDKN2A, and upon progression to PanIN-3, 75\% of tumors acquire a TP53 mutation, and $55 \%$ attain an SMAD4 mutation. ${ }^{11,13}$ Identifying these mutations and patients with high risk of developing these mutations is important as it is these patients who are at higher risk of developing cancer and recurrence.

\section{Case Presentation}

Clinical background

A 50-year-old Caucasian female, with no previous smoking history or pancreatic cancer family history, presented to our clinic for surgical evaluation of a biopsy-proven PDA noted at the junction of the body and tail of the pancreas. The patient had previously undergone a classic pancreaticoduodenectomy (Whipple) procedure with concurrent hemicolectomy 6 years prior (in 2011) for a T3N0M0 adenocarcinoma of the pancreatic head that had invaded the mesentery of the proximal transverse colon. After the 2011 surgery, pathology revealed a poorly differentiated PDA along with a PanIN grade 2. The final pathology showed negative surgical margins, positive perineural spread, and $0 / 33$ specimen lymph node involvement. From June 2012 to October 2012, the patient underwent and completed adjuvant chemotherapy with gemcitabine, capecitabine, and radiation at an outside hospital. She was carefully followed by her medical oncology team with serial CA 19-9 monitoring and abdominal MRIs on an ongoing basis. Due to her young age, in 2011, she underwent genetic screening and no germline mutations were identified. Since the time of the primary resection, the patient had been high functioning and healthy, with the exception of some problems of early satiety and recurrent cholangitis. These sequelae were attributed to close proximity of the gastrojejunostomy and hepaticojejunostomy, with possible reflux of intestinal contents up the afferent limb, all partially managed by diet changes.

In 2017, a biannual screening MRI with intravenous contrast showed a new pancreatic lesion measuring $2.3 \times 2.2 \mathrm{~cm}$ in the tail of the pancreas (Fig. 1). Esophagogastroduodenoscopy and endoscopic ultrasoundguided biopsy identified it as a poorly differentiated adenocarcinoma. From 2011 to 2017, she had had close followup with serial CA 19-9, and a measurement of this marker after identification of the lesion on MRI showed an elevation, which was confirmed on repeat testing (61 and $55 \mathrm{U} / \mathrm{mL}$; normal $<37 \mathrm{U} / \mathrm{mL}$ ). This was the first instance of two consecutive CA 19-9 measurements outside of the normal range since resection of the primary cancer 6 years prior. When the patient presented to our institution a few months later, the CA 19-9 had returned to normal at $32 \mathrm{U} / \mathrm{mL}$ (Fig. 2), and there was a moderate increase in CEA $(16.9 \mathrm{ng} / \mathrm{mL}$;

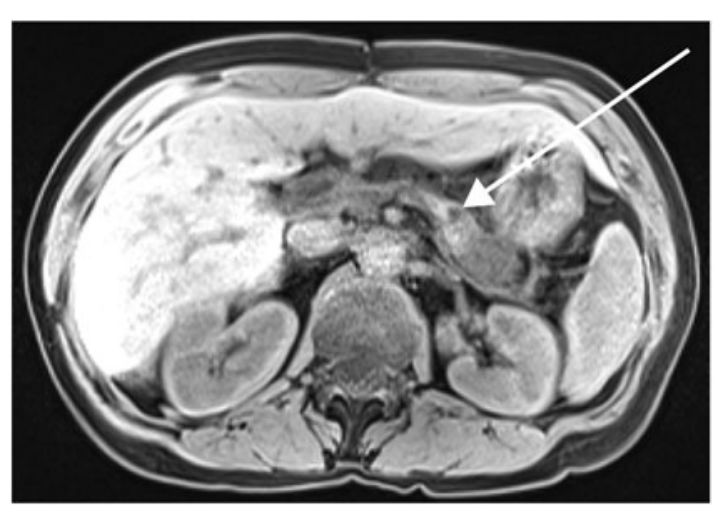

FIG. 1. MRI (axial T1 weighted) of the 2017 pancreatic mass. The image was read to show interval development of a mass within the pancreatic body-tail junction with restricted diffusion and hypoenhancement. The mass was measured to be $2.3 \times 2.2 \mathrm{~cm}$ (see arrow), and it was felt to focally encase the splenic artery and segmentally narrow the splenic vein. 


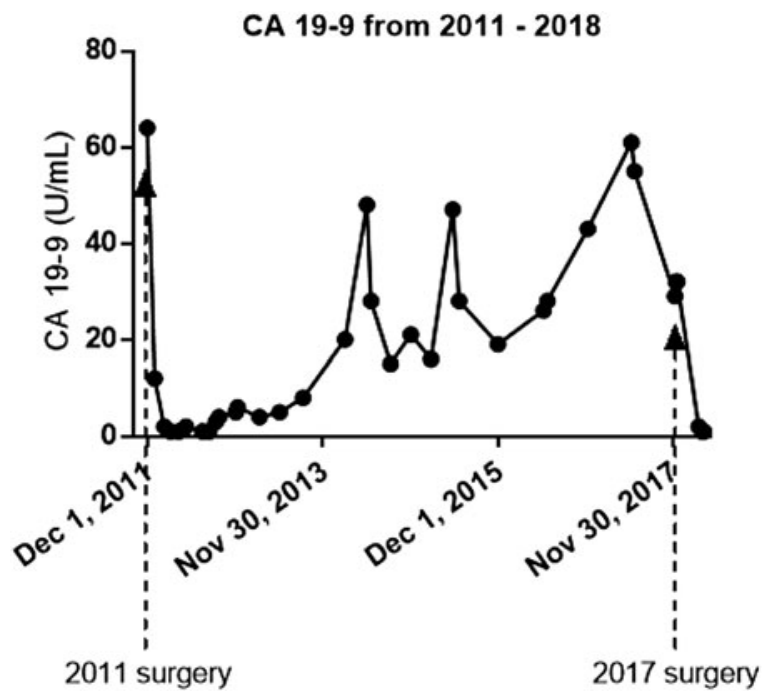

FIG. 2. Chronologic results of CA 19-9. Results are listed starting before the initial tumor resection in 2011 to identification of the new tumor in the pancreatic remnant in 2017.

normal $<3 \mathrm{ng} / \mathrm{mL}$ ). The patient received a second MRI of the abdomen and pelvis with contrast to identify distant disease, which showed the lesion to be confined to the pancreas. Along with the MRI of the abdomen, a CT of the chest was preformed, which showed no gross metastatic lesions. A completion pancreatectomy was scheduled with revision and lengthening of the jejunal limb proximal to the gastrojejunostomy to resect the tumor and treat her episodes of early satiety and recurrent cholangitis.

\section{Surgical intervention}

Intraoperatively, the patient's three Whipple anastomoses were noted to be grossly intact and there was no evidence of metastatic disease. First, the stomach was divided approximately two centimeters proximal to the prior gastrojejunostomy. The jejunostomy was closed, and gastrocolic and gastrosplenic ligaments were divided. The splenic artery was ligated, and the splenocolic ligament was divided. The spleen and pancreas were mobilized out of the retroperitoneum. The jejunum was divided between the pancreaticojejunostomy (PJ) and hepaticojejunostomy. The proximal jejunum, prior PJ, remaining pancreas, and spleen were removed. The tumor was noted to be grossly confined to the pancreas. The distal end of the stomach was delivered through the mesocolon and a retrocolic gastro- jejunostomy was undertaken $60 \mathrm{~cm}$ downstream from the hepaticojejunostomy. The anatomy before and after this operation is shown in Figure 3.

\section{Postoperative course}

The patient tolerated the procedure well and had an uncomplicated hospital course. The patient was closely followed postoperatively and did well. She completed two cycles of adjuvant chemotherapy with gemcitabine and capecitabine from January 2018 to April 2018, but did have some neutropenia at the end of her adjuvant therapy requiring pegfilgrastim. Her intermittent fevers, early satiety, and abdominal pain resolved after the surgery. She became an obligate insulin-dependent diabetic after the completion pancreatectomy procedure and now requires exogenous pancreatic enzymes to support her nutrient absorption.

\section{Pathology and molecular analysis}

The specimen was found to be consistent with a poorly differentiated invasive adenocarcinoma. Resection margins were negative, and 2 of 17 lymph nodes were positive for metastatic cancer. We sent representative slides of the patient's 2017 tumor and 2011 tumor to Perthera (McLean, VA) for next-generation sequencing (NGS) and histological analysis, which tested for mutations in a total of 315 genes and stained for various predictive biomarkers (Table 1). Both lesions showed the same mutations in KRAS (G12R), CDKN2A (splice site 151$1 \mathrm{G}$ to A), and TP53 (Y220C). In addition, her 2011 tumor had a mutation in $A C V R 1 B$ (S4) that was not present in the 2017 tumor, and the 2017 tumor had amplifications of $M Y C$ and mutant KRAS that were not present in the 2011 tumor (Table 1). Upon histological analysis, staining for MLH1, MSH2, MSH6, PMS2, pAKT, and HER2 was similar in both samples, but in the 2017 tumor, there was $60 \%$ increased staining for RRM1 and $20 \%$ increased staining for ERCC1, which changed the classification from low to high staining for ERCC1.

\section{Discussion}

A patient who has had surgical resection of pancreatic adenocarcinoma is at a high risk for recurrence, even with a seemingly adequate surgical intervention; more than $70 \%$ of patients with negative margins experience recurrence. ${ }^{6}$ The majority of these recurrences happen early, although there are reports of recurrence even after 5 years of disease-free survival. ${ }^{6}$ Some surgical specimen characteristics have been shown to influence 

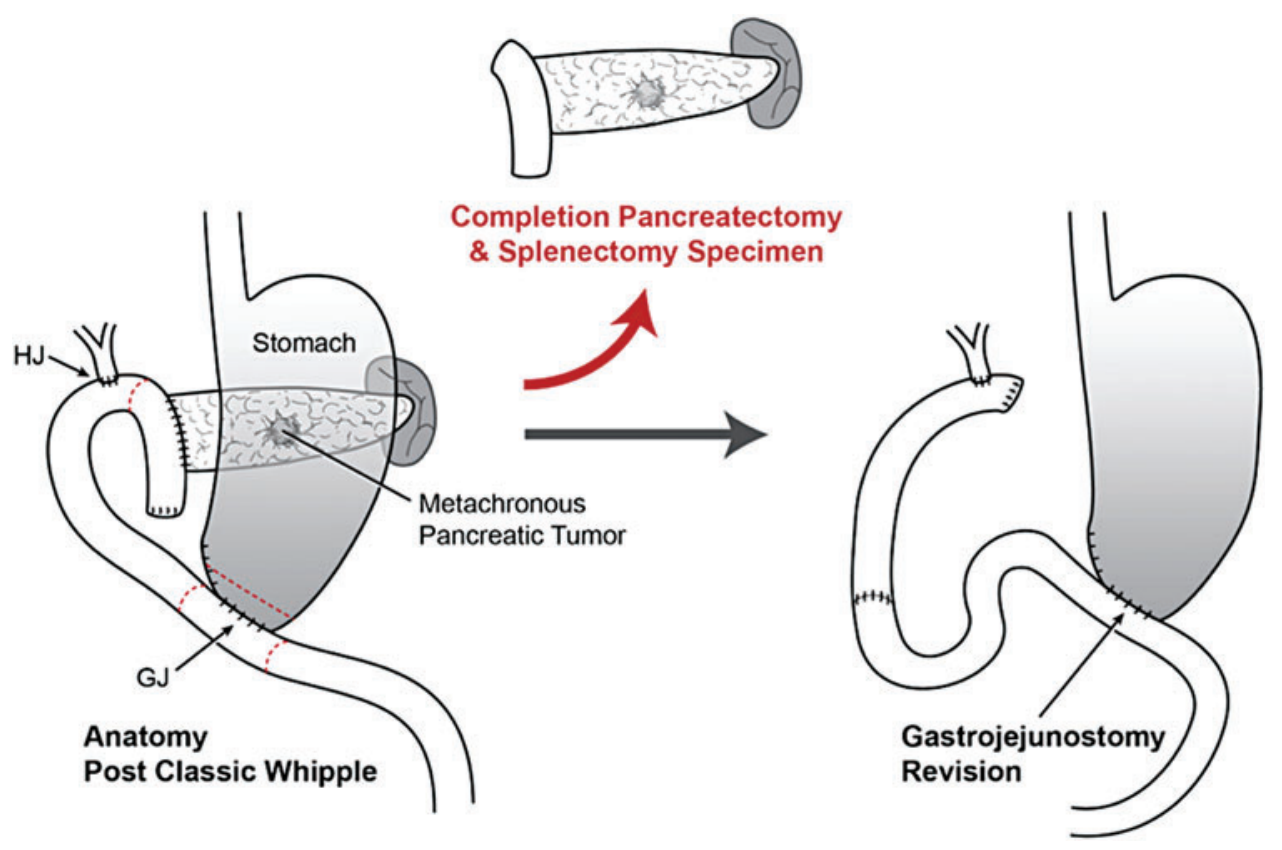

FIG. 3. Operative procedure summary; left depicting the patient's postclassic Whipple procedure anatomy (2011 surgery) and right depicting the patient's postrevision completion pancreatectomy anatomy (2017 surgery). Gastrojejunostomy was revised to address the patient's early satiety and cholangitis, which was thought to be due to a short afferent limb.

mortality, such as positive surgical margins, positive lymph node metastasis, large tumor size $(>3 \mathrm{~cm})$, and poor histological differentiation ${ }^{5,8}$; however, in resections that achieve R0 status, it seems that the presence of synchronously adjacent PanIN lesions in the surgical specimen (regardless of grade) does not influence outcomes. $^{14,15}$ There are no clear guidelines on how to screen for a recurrent PDA in postoperative pancreatic cancer patients. ${ }^{16}$ It is imperative to closely monitor these patients for signs of local and distant recurrence in the surgical bed as well as the liver, lungs, and peritoneal cavity, which are the most common sites of metastasis; this is most commonly achieved with CT. ${ }^{17}$
Our patient had a new lesion that was identified on MRI monitoring in 2017. Since our patient had a followup MRI of the abdomen and pelvis and CT of the chest that showed no metastatic disease, a completion pancreatectomy was planned, although a PET scan could have been utilized to rule out metastatic disease as well. For patients with no evidence of distant metastatic disease and an apparent pancreas-confined lesion, as was the case with our patient, resection of the new lesion can be safe and effective. 9,10

It is often unclear if a new lesion that occurs in an organ after extended cancer-free survival represents a recurrent lesion or a second primary/metachronous

Table 1. Summary of Next-Generation Sequencing on Both 2011 and 2017 Tumor Samples

\begin{tabular}{|c|c|c|c|c|c|c|}
\hline \multicolumn{3}{|c|}{2011 Sample } & \multicolumn{4}{|c|}{2017 Sample } \\
\hline $\begin{array}{l}\text { Genes } \\
\text { tested }\end{array}$ & Mutation & $\begin{array}{l}\text { Mutation allele } \\
\text { frequency (\%) }\end{array}$ & $\begin{array}{l}\text { Gene } \\
\text { tested }\end{array}$ & Mutation/amplification & $\begin{array}{l}\text { Mutation allele } \\
\text { frequency (\%) }\end{array}$ & $\begin{array}{l}\text { Amplification } \\
\text { copy number }\end{array}$ \\
\hline KRAS & $\mathrm{G} 12 \mathrm{R}$ & 15.3 & KRAS & $\mathrm{G} 12 \mathrm{R}$ & 88.3 & 38 \\
\hline ACVR1B & S4 & 14.9 & ACVR1B & (No mutation identified, & type) & \\
\hline CDKN2A & Splice Site $151-1 \mathrm{G}>\mathrm{A}$ & 14.4 & CDKN2A & Splice site $151-1 \mathrm{G}>\mathrm{A}$ & 52 & \\
\hline TP53 & Y220C & 10.3 & TP53 & Y220C & 44 & \\
\hline MYC & \multicolumn{2}{|c|}{ (No mutation identified, wild type) } & MYC & Amplification & & 76 \\
\hline
\end{tabular}


cancer. The concept of a field defect was first introduced in 1957 by Slaughter et al. and describes an underlying genetic defect of an organ or developmental field that predisposes that tissue to cancer. ${ }^{18}$ Subsequently, it has been shown that field defects can cause shared genetic mutations of large patches of tissue and, in extreme cases, entire organs. ${ }^{19}$ While initially described in oral epithelial cancers, the concept of field defects has been reproduced in many different organ systems, including the pancreas. ${ }^{20}$ Field defects have been implicated in cases of second primary tumors (also referred to as second field tumors). ${ }^{20}$ Second primary tumors tend to occur at sites distant to the primary resected surgical site, as opposed to recurrences that are adjacent to the surgical scar. ${ }^{20}$ When assessing whether a lesion is a second primary or recurrent lesion, it is important to remember that genetically the recurrent tumor should be near identical to the original. In the case of a second primary tumor, there can be new mutations that are unique to the second primary tumor, and mutations present in the primary tumor may not be represented in the second tumor. ${ }^{19,20}$

Due to identification of the patient's new lesion many years following the initial procedure, which had negative margins, and the fact that the mass was not noted at the margins of the prior procedure, it was our clinical suspicion that this may be a case of a metachronous cancer. Our genetic testing confirmed that the new tumor in 2017 had similar genetic mutations in KRAS, TP53, and CDKN2A compared with the 2011 cancer, but the mutation in $A C V R 1 B$ was not present in the 2017 sample, and mutant KRAS (copy number 38) and MYC (copy number 76) amplifications were only present in the 2017 sample. Without further and more sophisticated genetic testing, we cannot determine precisely if the new tumor represents a second primary tumor or a recurrent tumor.

KRAS and MYC have been extensively studied with regard to cancer. KRAS mutations are present in over $90 \%$ of pancreatic cancers and are noted as being the earliest genetic alterations in majority of these cancers. ${ }^{12}$ KRAS encodes a proto-oncogene GTPase, which (when mutated) results in constitutively activated signaling pathways leading to sustained proliferation and antiapoptosis. ${ }^{21} \mathrm{MYC}$ is a transcription factor and is implicated in over 450,000 American patients with cancers per year. ${ }^{22}$ MYC acts as a sensor, integrating cellular signals and ultimately affecting cellular responses, including differentiation, survival, and pluripotency. ${ }^{23}$ MYC has a short half-life in the cell, estimated to be
20-30 $\mathrm{min}$, and its production and degradation are tightly regulated by transcription factors, ubiquitin proteasome degradation, and micro-RNA. ${ }^{24-26}$ Mutations that result in amplification or overexpression of $M Y C$ result in tumorigenesis. ${ }^{23,27}$

$M Y C$ and $c-M Y C$ deregulation is common in PDA, with $\sim 30 \%$ of primary and metastatic tumors showing MYC amplification. ${ }^{28}$ In a small sample size, Schleger et al. were able to identify two patients with high MYC amplification in the metastatic site when compared with the primary tumor, which had low MYC amplification. $^{28}$ There was strong correlation between MYC amplification and protein expression and tumor grade in this population as well, demonstrating its role in PDA progression.

Although there is limited literature published in regard to $M Y C$ and KRAS amplifications in pancreatic metachronous tumors, in breast cancer, $c-M Y C$ amplification is associated with tumor progression, earlier tumor relapse, and worse overall survival. ${ }^{29}$ In patients with recurrent breast cancer tumors with $c-M Y C$ amplification, it has been found that half of these patients with breast cancer lose their hormone receptor expression and overall have shorter recurrence-free survival when compared with patients with normal $c-M Y C$ status. $^{29}$ It was also found that $M Y C$ amplification is a risk factor for developing chemoresistance in patients with breast cancer, except for chemotherapeutics such as doxorubicin and cyclophosphamide. ${ }^{29}$ In endometrial cancer, KRAS amplification has been seen in $18 \%$ of metastatic lesions compared with only $3 \%$ in primary tumors $(p<0.001)$, and amplification status correlates with poor outcome, higher grade, and lost hormone receptor expression. ${ }^{30}$

In our patient, it is possible that the patterns of mutations and amplifications seen in the 2011 and 2017 tumors are due to a field defect or genetic vulnerability involving the entire pancreas. NGS analysis of the two tumors showed an amplification of MYC with a copy number of 76 in the 2017 tumor, which was not present in the 2011 tumor, along with a significant mutant KRAS amplification. Furthermore, it stands to reason that the shared KRAS, TP53, and CDKN2A mutations were the direct result of a field defect of the pancreas, while MYC amplification was not.

Another possibility that explains these genetic similarities and differences is the characteristic of tumor heterogeneity. Three identical driver mutations were present in both of the patient's lesions, which may be indicative of a recurrence/metastasis. In this case, loss 
of the $A C V R 1 B$ mutation can be reconcilable by tumor heterogeneity, or a later selected mutation, or simply a passenger mutation. PDA, in general, has a high degree of genetic heterogeneity, with varying degrees of mutations from one area of the tumor to the other, which can also influence chemotherapy response. ${ }^{31,32}$ Over the time course of tumor progression, separate cancerous foci can progress to PDA (i.e., clonal progression), but due to varying degrees of genetic heterogeneity, each clone can have a different genetic makeup even though they all originated from the same original clone. $^{31}$ Thus, it is possible that due to selection and sampling bias during NGS, the MYC and KRAS amplifications were not identified, but were present in both the 2011 and 2017 samples. In addition, these amplifications can occur purely due to the 6-year time period between tumor developments and may be an example of normal PDA progression over time. In an article by Yachida et al., genetic analysis of a patient's primary and metastatic tumors showed an average of $64 \%$ of mutations to be due to founder mutations (present in the original clone) and an average of $36 \%$ of mutations to be due to progressor mutations (not present in the original clone) with clonal evolution and metastasis. ${ }^{32}$ These progressor mutations, such as MYC and KRAS amplification in our case, were not found in the parental clone and can be considered to either be passenger or driver mutations and could have possibly contributed to development of the 2017 tumor. Many hypotheses can be made in regard to the reason why the NGS is different between the 2011 and 2017 samples, so their results should be taken in context. One could seek to elucidate the true nature of the patient's second lesion with additional tests to demonstrate clonality, such as single-nucleotide polymorphism arrays or X-gene imprinting, although this was not feasible in our case due to limited amount of viable specimen present.

Although the 2011 sample had negative resection margins and lymph node status, microdissemination could have occurred and led to the 2017 tumor development, favoring the argument that the 2017 tumor is a result of recurrence and not a metachronous tumor. As highlighted in this case, the use of NGS is a tool and should be used as an adjunct in diagnosis, but it does not serve as a stand-alone test to distinguish between recurrence and metachronous lesions. This case report brings to light the importance of clinical correlation when interpreting genetic testing results.

Although the data gathered from genetic testing are invaluable, we would like to discuss some logistical chal- lenges that we encountered during this process. First, when it was identified that this patient was rediagnosed with PDA in 2017, it was difficult to obtain the 2011 tumor sample for comparison as over the past 6 years the patient had received care at multiple different hospitals. Second, the 2011 tumor slides had a heavy necrotic burden, so many of them could not be used for NGS, and fragments of the tumor from multiple slides were needed to allow for enough usable DNA. Third, lack of coordination between different institutions' medical records, pathology, and insurance approval for testing also led to delays in obtaining sequencing results. In the end, the samples were able to be processed, but it is important to remember that without assistance from members in all these departments and institutions, these genetic findings would be not have been successfully identified.

\section{Conclusion}

We present a case of a 50-year-old female who has experienced two instances of PDA at a young age. With the use of NGS, we were able to perform genomic profiling of both lesions, which identified both retained and unique mutations between the two samples. With the limited NGS data we obtained, we cannot definitively conclude the molecular etiology of this secondary tumor. Although the distinction between recurrent and metachronous lesions can be vague, we can confidently write that common genetic drivers between the 2011 and 2017 tumors are completely identical. Therefore, in a hypothetical case where these genetic drivers are actionable, they could have clinical value in the future as potential predictive and/or resistance markers for next-line therapy.

\section{Acknowledgments}

The authors would like to thank the patient and her family for participating and allowing them to write this report about her case from 2011 to 2017. The authors would also like to thank Perthera, Inc., in collaboration with Foundation Medicine, Inc. (Morrisville, NC), and NeoGenomics Laboratories, Inc. (Viejo, CA), for NGS and histological analysis of the 2011 tumor and the 2017 tumor free of cost to the patient. The work in this article was supported by the NIH/ NCI Cancer Center Support Grant P30 CA056036.

\section{Author Disclosure Statement}

J.R.B. is on the Scientific Advisory Board of Perthera, Inc.; M.J.P. is in an employment or leadership position with Perthera, Inc.; and C.J.Y., T.M.B., T.D., A.S., H.T., A.B.G., and S.C. have no competing financial interests. 


\section{References}

1. American Cancer Society. Cancer Facts and Figures 2013. American Cancer Society: Atlanta; 2013. Available at https://www.cancer.org/ content/dam/cancer-org/research/cancer-facts-and-statistics/annualcancer-facts-and-figures/2013/cancer-facts-and-figures-2013.pdf Accessed on May 3, 2018.

2. DiMagno EP, Reber HA, Tempero MA. AGA technical review on the epidemiology, diagnosis, and treatment of pancreatic ductal adenocarcinoma. Gastroenterology. 1999;117:1464-1484.

3. Becker $A E$, Hernandez $Y G$, Frucht $H$, et al. Pancreatic ductal adenocarcinoma: risk factors, screening, and early detection. World J Gastroenterol. 2014;20:11182-11198.

4. Gillen S, Schuster T, Meyer Zum Büschenfelde C, et al. Preoperative/ neoadjuvant therapy in pancreatic cancer: a systematic review and meta-analysis of response and resection percentages. PLoS Med. 2010;7: e1000267.

5. Benassai G, Mastrorilli M, Quarto $G$, et al. Factors influencing survival after resection for ductal adenocarcinoma of the head of the pancreas. J Surg Oncol. 2000;73:212-218.

6. Schnelldorfer T, Ware AL, Sarr MG, et al. Long-term survival after pancreatoduodenectomy for pancreatic adenocarcinoma: is cure possible? Ann Surg. 2008;247:456-462.

7. Conroy T, Hammel P, Hebbar M, et al. Unicancer GI PRODIGE $24 / C C T G$ PA.6 trial: a multicenter international randomized phase III trial of adjuvant mFOLFIRINOX versus gemcitabine (gem) in patients with resected pancreatic ductal adenocarcinomas. J Clin Oncol. 2018;36(18_suppl): LBA4001.

8. Benassai G, Mastrorilli M, Quarto G, et al. Survival after pancreaticoduodenectomy for ductal adenocarcinoma of the head of the pancreas. Chir Ital. 2000:52:263-270.

9. Lavu H, Nowcid LJ, Klinge MJ, et al. Reoperative completion pancreatectomy for suspected malignant disease of the pancreas. J Surg Res. 2011; 170:89-95.

10. Miyazaki M, Yoshitomi H, Shimizu H, et al. Repeat pancreatectomy for pancreatic ductal cancer recurrence in the remnant pancreas after initial pancreatectomy: is it worthwhile? Surgery. 2014;155: 58-66.

11. Bryant $\mathrm{KL}$, Mancias JD, Kimmelman AC, et al. KRAS: feeding pancreatic cancer proliferation. Trends Biochem Sci. 2014;39:91-100.

12. Kanda $\mathrm{M}$, Matthaei $\mathrm{H}, \mathrm{Wu}$ J, et al. Presence of somatic mutations in most early-stage pancreatic intraepithelial neoplasia. Gastroenterology. 2012; 142:730-733.e9.

13. Hezel AF, Kimmelman AC, Stanger BZ, et al. Genetics and biology of pancreatic ductal adenocarcinoma. Genes Dev. 2006;20: 1218-1249.

14. Matthaei $\mathrm{H}$, Hong S-M, Mayo SC, et al. Presence of pancreatic intraepithelial neoplasia in the pancreatic transection margin does not influence outcome in patients with R0 resected pancreatic cancer. Ann Surg Oncol. 2011;18:3493-3499.

15. Yu D-Y, Yu Y-D, Kim W-B, et al. Clinical significance of pancreatic intraepithelial neoplasia in resectable pancreatic cancer on survivals. Ann Surg Treat Res. 2018;94:247-253.

16. Sheffield KM, Crowell KT, Lin Y-L, et al. Surveillance of pancreatic cancer patients after surgical resection. Ann Surg Oncol. 2012;19: 1670-1677.

17. Van den Broeck A, Sergeant G, Ectors N, et al. Patterns of recurrence after curative resection of pancreatic ductal adenocarcinoma. Eur J Surg Oncol. 2009;35:600-604.

18. Slaughter DP, Southwick HW, Smejkal W. Field cancerization in oral stratified squamous epithelium. Clinical implications of multicentric origin. Cancer. 1953;6:963-968.

19. Braakhuis BJM, Tabor MP, Kummer JA, et al. A Genetic explanation of slaughter's concept of field cancerization: evidence and clinical implications. Cancer Res. 2003;63:1727-1730.

20. Dakubo GD, Jakupciak JP, Birch-Machin MA, et al. Clinical implications and utility of field cancerization. Cancer Cell Int. 2007;7:2.

21. Pylayeva-Gupta Y, Grabocka E, Bar-Sagi D. RAS oncogenes: weaving a tumorigenic web. Nat Rev Cancer. 2011;11:761-774.

22. Hessmann E, Schneider G, Ellenrieder V, et al. MYC in pancreatic cancer: novel mechanistic insights and their translation into therapeutic strategies. Oncogene. 2016;35:1609-1618.
23. Conacci-Sorrell M, McFerrin L, Eisenman RN. An overview of MYC and its interactome. Cold Spring Harb Perspect Med. 2014;4:a014357.

24. Farrell AS, Sears RC. MYC degradation. Cold Spring Harb Perspect Med. 2014:4:a014365.

25. Hann SR, Eisenman RN. Proteins encoded by the human c-myc oncogene: differential expression in neoplastic cells. Mol Cell Biol. 1984;4:2486-2497.

26. Psathas JN, Thomas-Tikhonenko A. MYC and the art of microRNA maintenance. Cold Spring Harb Perspect Med. 2014;4:a014175.

27. Farrell AS, Joly MM, Allen-Petersen BL, et al. MYC regulates ductalneuroendocrine lineage plasticity in pancreatic ductal adenocarcinoma associated with poor outcome and chemoresistance. Nat Commun. 2017 8:1728.

28. Schleger C, Verbeke C, Hildenbrand R, et al. c-MYC activation in primary and metastatic ductal adenocarcinoma of the pancreas: incidence, mechanisms, and clinical significance. Mod Pathol. 2002;15:462-469.

29. Aulmann S, Adler N, Rom J, et al. c-myc amplifications in primary breast carcinomas and their local recurrences. J Clin Pathol. 2006;59: 424-428.

30. Birkeland E, Wik E, Mjøs S, et al. KRAS gene amplification and overexpression but not mutation associates with aggressive and metastatic endometrial cancer. Br J Cancer. 2012;107:1997-2004.

31. Bardeesy N, DePinho RA. Pancreatic cancer biology and genetics. Nat Rev Cancer. 2002;2:897-909.

32. Yachida S, Jones S, Bozic I, et al. Distant metastasis occurs late during the genetic evolution of pancreatic cancer. Nature. 2010;467:1114-1117.

Cite this article as: Bauer TM, Dhir T, Strickland A, Thomsett $\mathrm{H}$, Goetz AB, Cannaday S, Brody JR, Pishvaian MJ, Yeo CJ (2018) Genetic drivers of pancreatic cancer are identical between the primary tumor and a secondary lesion in a long-term ( $>5$ years) survivor after a Whipple procedure, Journal of Pancreatic Cancer 4:1, 81-87, DOI: 10.1089/ pancan.2018.0015.
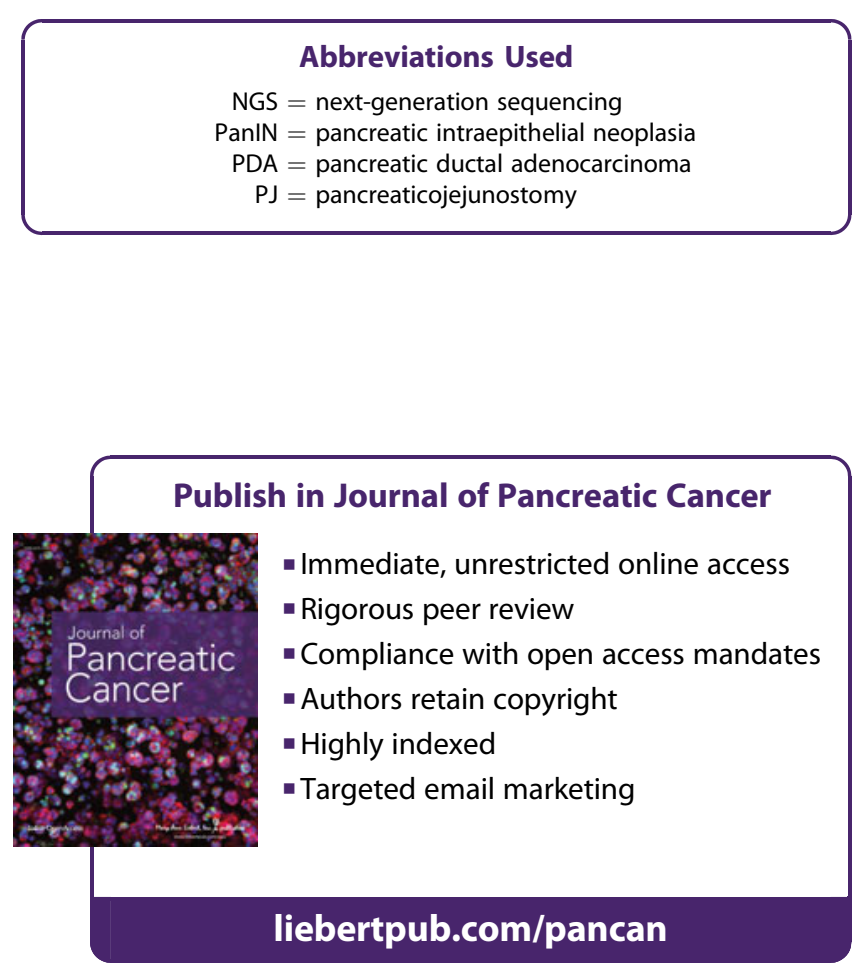